Nervenarzt 2021 · 92:540-547 https://doi.org/10.1007/s00115-021-01094-0 Angenommen: 2. Februar 2021 Online publiziert: 26. März 2021 (c) Springer Medizin Verlag $\mathrm{GmbH}$, ein Teil von Springer Nature 2021

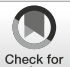

Helmar C. Lehmann' • Benedikt Schoser ${ }^{2}$ Gilbert Wunderlich ${ }^{1,5} \cdot$ Peter Berlit ${ }^{3}$. Gereon R. Fink ${ }^{1,4}$

${ }^{1}$ Klinik und Poliklinik für Neurologie, Universitätsklinikum Köln, Köln, Deutschland

${ }^{2}$ LMU Klinikum, Friedrich-Baur-Institut, Neurologische Klinik und Poliklinik, Ludwig-MaximiliansUniversität München, München, Deutschland

${ }^{3}$ Deutsche Gesellschaft für Neurologie (DGN), Berlin, Deutschland

${ }^{4}$ Institut für Neurowissenschaften (INM-3), Forschungszentrum Jülich, Jülich, Deutschland

${ }^{5}$ Zentrum für Seltene Erkrankungen, Universitätsklinikum Köln, Köln, Deutschland

\title{
Neuromuskuläre Komplikationen einer SARS-CoV-2-Infektion - Teil 1: periphere Nerven
}

muskuläre Komplikationen einer SARSCoV-2-Infektion - Teil 2: Erkrankungen der Muskulatur" ist es daher, die bisherige Literatur hinsichtlich möglicher neuromuskulärer Manifestationen von COVID-19 einzuordnen.

\section{COVID-19} "Gesundheitsnotstand von internationaler Bedeutung". Weltweit haben sich im Rahmen der Pandemie über 100 Mio. Menschen infiziert, und etwa 2 Mio. Menschen sind mit oder an COVID-19 verstorben (Stand Januar 2021, Quelle: Johns Hopkins Universität). COVID-19 als weltweites Ereignis von höchster medizinscher Relevanz dominiert auch die wissenschaftliche Literatur der letzten Monate. Eine Abfrage der Literaturdatenbank „PubMed“ ergibt, dass in den Monaten Februar bis Oktober 2020 etwa $7 \%$ aller aufgeführten wissenschaftlichen Artikel das Schlagwort COVID19 enthalten. Bei Artikeln, die unter dem MeSH-Schlagwörtern „diseases of peripheral nervous system“ und "neuromuscular diseases" summiert sind, wächst der Anteil von COVID-19-Artikeln sogar auf über $20 \%$ an, während die absolute Anzahl neurologischer Artikel deutlich abnimmt. Dieser Publikationstrend suggeriert, dass eine relevante Assoziation zwischen COVID-19 und neurologischen bzw. speziell neuromuskulären Erkrankungen existiert. Ziel dieses Artikels und des Artikels „Neuro-
Erste und häufigste Symptome von COVID-19 sind Fieber, unproduktiver Husten und Dyspnoe aufgrund der durch das Virus verursachten Pneumonie. Anhand der bisherigen Erfahrungen kann der typische Krankheitsverlauf von COVID-19 in verschiedene Phasen eingeteilt werden (• Abb. 1). Im unteren Abschnitt von $\bullet$ Abb. 1 sind die Zeiträume aufgetragen, in denen neuromuskuläre Symptome und Erkrankungen beschrieben wurden.

Nach häufig asymptomatischer Infektion (1. Phase) wird SARS-CoV-2 im Nasen-Rachen-Raum aktiv repliziert. Bei Patienten, die in die 2. (pulmonale oder pneumovaskuläre) Phase eintreten, entwickeln sich grippeähnliche Symptome wie Fieber, Husten und Inappetenz. Das Fieber hält im Mittel 12 bis 13 Tage an, während der Husten etwa 19 Tage besteht. Eine Dyspnoe beginnt häufig am Tag 5 bis 7 nach Symptombeginn. Eine intensivpflichtige Ateminsuffizienz tritt etwa ab dem 10. Tag ein. In der späten, hyperinflammatorischen Erkrankungsphase (3. Phase) stehen immunologische Pro- zesse im Vordergrund, eine Virusreplikation ist meist nicht mehr nachweisbar. Pathogenetisch kommt es in dieser Phase häufig zu einer massiven Entzündungsreaktion mit klinischer Verschlechterung und Multiorganversagen.

Verschiedene Szenarien sind postuliert worden, auf welche Weise eine SARS-CoV-2-Infektion neuromuskuläre Erkrankungen hervorrufen oder zumindest aggravieren kann [12].

\section{》) COVID-19 kann periphere Nerven und Muskeln schädigen}

Mit Abstand am häufigsten wird in der Literatur über eine Assoziation einer SARS-CoV-2-Infektion mit Störungen der Funktion peripherer Nerven (einschließlich Hirnnerven) und Schädigung der Muskulatur berichtet. Prinzipiell ist auch vorstellbar, dass eine SARS-CoV-2Infektion den Verlauf neuromuskulärer Erkrankungen aggravieren oder eine klinische Erstmanifestation triggern kann. Diesbezüglich finden sich allerdings bisher nur wenige Fallberichte, z. B. bei einer Myasthenia gravis [38 39 45]. Zudem kann die zumindest zeitweise angewandte Therapie mit Hydroxychloroquin toxische Neuro- und Myopathien verursachen [4446] bzw. eine Myasthenia gravis verschlechtern [23]. Eine beeinträchtigte Lungenfunktion im Rahmen von Motoneuronerkrankungen, heredi- 
Hier steht eine Anzeige.

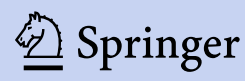




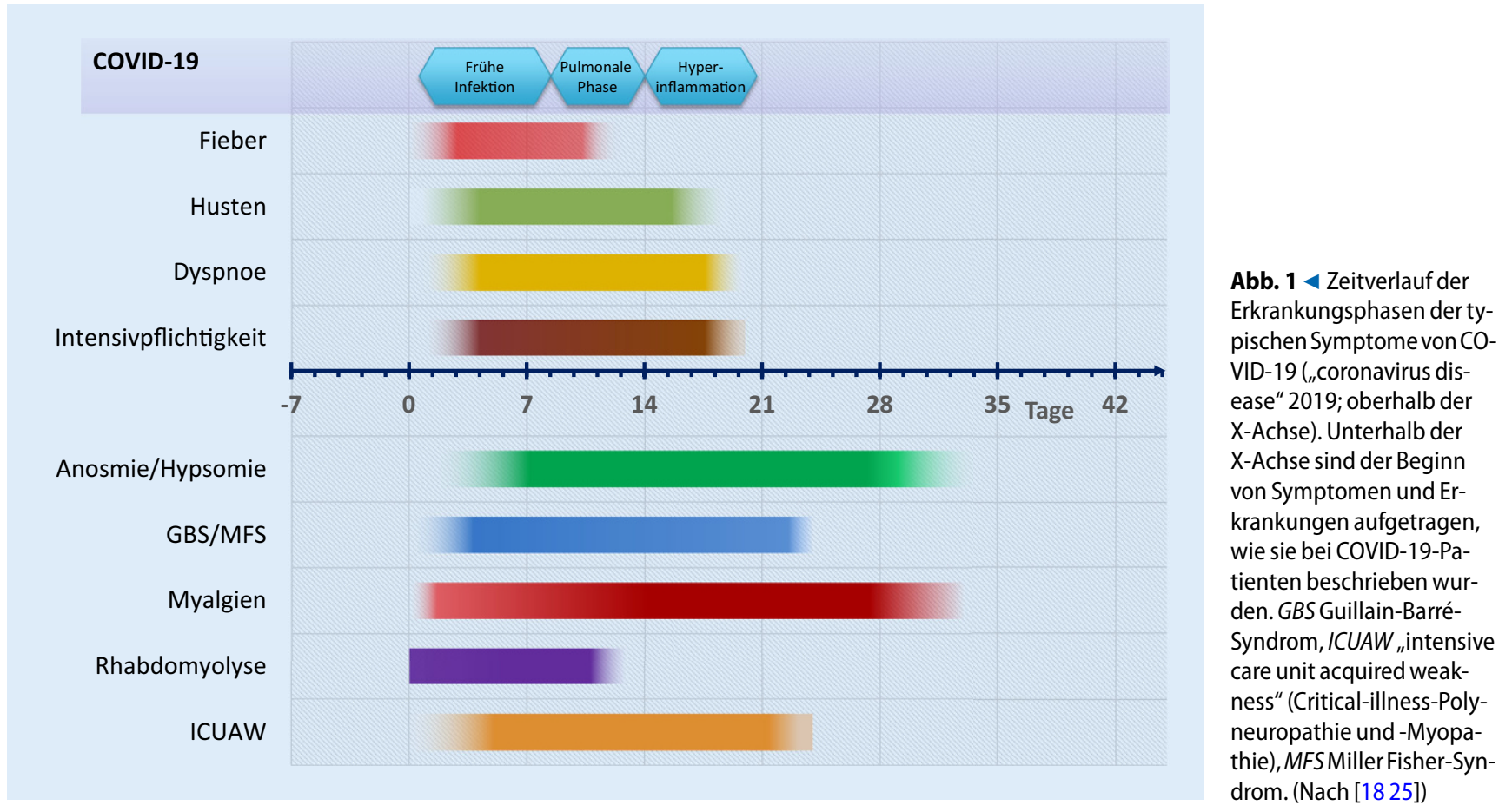

tären Neuropathien, Muskeldystrophien oder Myopathien könnte zudem ein Risiko für einen schweren Verlauf von COVID-19 darstellen. Eine Fallserie berichtet beispielsweise vom letalen Ausgang einer COVID-19-Infektion bei drei Patienten mit myotoner Dystrophie Typ 1 und bereits zuvor eingeschränkter Atemfunktion mit Notwendigkeit nichtinvasiver Heimbeatmung [9].

Dass neuromuskuläre Erkrankungen aufgrund respiratorischer Dysfunktion oder einer immunsuppressiven Therapie (z. B. bei der chronisch inflammatorischen demyelinisierenden Polyradikuloneuropathie [26], vaskulitischen Neuropathien [42], Myositiden [41]) prinzipiell einen Risikofaktor für eine erhöhte Morbidität und Mortalität bei einer SARS-CoV-2-Infektion darstellen, ist allerdings nicht belegt. Studien aus China, Europa und den USA konnten zwar Komorbiditäten wie Diabetes mellitus und chronische Lungenerkrankungen als Risikofaktoren für eine erhöhte Mortalität bei COVID-19 identifizieren, in diesen Studien fanden sich jedoch keine Hinweise für eine erhöhte Hospitalisierungsrate von Patienten mit neuromuskulären Erkrankungen [6 54].

\section{SARS-CoV-2 als Auslöser neuromuskulärer Symptome und Erkrankungen}

In den vergangenen Monaten wurden folgende Symptome und Erkrankungen der peripheren Nerven und Muskulatur während oder nach einer COVID-19-Erkrankung beschrieben:

- Anosmie, Ageusie und andere Hirnnervenausfälle (N. facialis, Augenmuskelnerven),

- Guillain-Barré-Syndrom/Miller Fisher-Syndrom,

- Armplexopathie (neuralgische Schulteramyotrophie).

Auch muskuläre Erkrankungen und Störungen der neuromuskulären Transmission wie Myasthenia gravis, Myalgien, Myositiden, Rhabdomyolyse und „intensive care unit acquired weakness“ (ICUAW) wurden beschrieben, die im zweiten Teil des Reviews (Neuromuskuläre Komplikationen einer SARS-CoV2-Infektion - Teil 2: Erkrankungen der Muskulatur) diskutiert werden.

\section{Anosmie und Ageusie}

Die Beeinträchtigung des Geruchs- oder des Geschmacksinns stellt ein typisches
Frühsymptom einer COVID-19-Infektion dar, was in mehr als 20 Studien bestätigt wurde (Übersicht in [34]).

\section{Merke}

Akute Anosmie und Ageusie ist so häufig bei SARS-COV-2-Infektionen, dass es von vielen als pathognomonische Symptomkonstellation angesehen wird (C. Lehmann, persönl. Kommunikation).

Allerdings kommen Studien zu unterschiedlichen Ergebnissen hinsichtlich der Häufigkeit. Diese lag für Hyposmie/ Anosmie zwischen 11,9 und $85 \%$ und für Dysgeusie/Ageusie zwischen 15,3 und $88,8 \%$ in europäischen Untersuchungen [16 25], in chinesischen Studien bei etwa $20 \%$ [32]. Es ist aktuell unklar, ob es sich hier um echte geographische Unterschiede handelt oder ob die unterschiedlichen Inzidenzen auf eine Variabilität der Methoden zurückzuführen sind, da sich die Studien bez. Design, Datenerhebung etc. erheblich unterscheiden. Zudem wird in den meisten Studien nicht zwischen (I) der Funktion des Geschmacksorgans, das lediglich die Qualitäten süß, salzig, sauer und bitter wahrnimmt, und (II) der Funktion der Riechschleimhaut, die die "Geschmacksempfindung“ und 
"Aromen“ wahrnimmt, unterschieden. Erstere wird über Geschmacksknospen im Bereich der seitlichen Zunge, die vom N. facialis bzw. N. glossopharyngeus innerviert werden, wahrgenommen. Die bisher nur vereinzelt beschriebene Affektion motorischer Anteile des N. facialis bzw. des N. glossopharyngeus legt nahe, dass die Symptome Hyposmie/Anosmie/ Dysgeusie/Ageusie auf eine Dysfunktion der Riechschleimhaut und der Nn. olfactorii zurückzuführen sind.

In der Magnetresonanztomographie (MRT) kann während der Anosmie ein transiente ödematöse Schwellung der Bulbi olfactorii beobachtet werden [24]. Bei prolongierter olfaktorischer Dysfunktion geht diese mit eine Atrophie der Bulbi olfactorii im MRT einher [5 51].

\section{》) Geruchs-/Geschmacks- symptome korrelieren nicht mit der Schwere von COVID-19}

Nach einer aktuellen Untersuchung der Universitätsklinik Köln [31] treten die Symptome einer Geruchs- oder Geschmackssinnstörung in der Mehrzahl der Fälle gemeinsam auf. Bei 3 von $4 \mathrm{~Pa}$ tienten kommt es zu einer Beeinträchtigung des Geruchssinns (Hyposmie), während bei fast $70 \%$ eine verminderte Geschmacksempfindung (Hypogeusie) vorhanden war. Die Symptome treten in der Studie von Luers und Mitarbeitern in der Regel nach anderen Symptomen auf, im Durchschnitt am 4. Tag nach Erkrankungsbeginn und gingen oft mit einer Rhinorrhö einher. Dies deckt sich mit Beobachtungen aus europäischen Nachbarländern, in denen die Symptome nur bei etwa jedem 10. Patienten vor Beginn anderer Symptome $(11,8 \%)$ und meistens danach $(65,4 \%)$ oder gleichzeitig $(22,8 \%)$ mit den Allgemeinsymptomen auftraten [25]. Das Vorhandensein von Geruchs-/Geschmackssymptomen korrelierte nicht mit der Krankheitsschwere von COVID-19 und hat auch keinen prognostischen Wert für den Schweregrad von COVID-19 [52].

Prinzipiell kann eine olfaktorische Dysfunktion aufgrund einer lokalen Störung der mechanischen Barriere

Nervenarzt 2021 · 92:540-547 https://doi.org/10.1007/s00115-021-01094-0

๑) Springer Medizin Verlag GmbH, ein Teil von Springer Nature 2021

\section{H. C. Lehmann · B. Schoser · G. Wunderlich · P. Berlit · G. R. Fink}

\section{Neuromuskuläre Komplikationen einer SARS-CoV-2-Infektion - Teil 1: periphere Nerven}

\section{Zusammenfassung}

In den letzten Monaten sind im Zusammenhang mit einer durch das "severe acute respiratory syndrome coronavirus 2" (SARSCoV-2) ausgelösten Lungenerkrankung COVID-19 ("Coronavirus disease 2019") verschiedene Störungen und Erkrankungen der peripheren Nerven (einschließlich Hirnnerven) und der Muskulatur beschrieben worden. Im ersten Teil unserer Übersicht diskutieren wir den aktuellen Stand einer möglichen Assoziation einer SARS-CoV-2Infektion mit Affektionen peripherer Nerven (inklusive Hirnnerven). So wurden u. a. eine Anosmie und Ageusie, motorische Hirnnervenbeteiligung und Guillain-Barré-Syndrome (GBS) in zeitlichem Zusammenhang mit einer SARS-CoV-2-Infektion beschrieben. Mehrere Studien konnten zeigen, dass Anosmie und
Ageusie häufige Symptome einer SARSCoV-2-Infektion sind. Im Gegensatz dazu sind andere Hirnnervenausfälle bisher nur vereinzelt beschrieben worden. Eine Reihe von Fallberichten und Fallserien legt einen kausalen Zusammenhang zwischen einer SARS-CoV-2-Infektion und GBS nahe, epidemiologische Evidenz hierfür ist aber ausstehend.

\section{Schlüsselwörter}

Polyradikuloneuropathie [C10.114.750] · Neuromuskuläre Manifestationen [C10.597.613] Neuromuskuläre Erkrankungen [C10.668] · Erkrankungen des peripheren Nervensystems [C10.668.829] · Molekulares Mimikry [G02.111.560]

\section{Neuromuscular complications of SARS-CoV-2 infections-Part 1: peripheral nerves}

Abstract

In recent months various disorders and diseases of the peripheral nerves (including cranial nerves) and the musculature have been described in association with the pulmonary disease coronavirus disease 2019 (COVID-19) caused by the severe acute respiratory syndrome coronavirus 2 (SARS-CoV-2). In the first part of our review the current knowledge about a potential association of a SARS-CoV- 2 infection with dysfunction and diseases of cranial and peripheral nerves is discussed. Anosmia, ageusia, motor cranial nerve involvement and Guillain-Barré syndrome (GBS) were described in a temporal association with a SARS-CoV-2 infection. Several studies could show that anosmia and ageusia were frequent symptoms of a SARS-CoV-2 infection. In contrast the failure of other cranial nerves has so far only been sporadically described. A number of case reports and case series indicate a causal association between a SARSCoV-2 infection and GBS but epidemiological evidence is still lacking.

\section{Keywords}

Polyradiculoneuropathy [C10.114.750] . Neuromuscular manifestations [C10.597.613] Neuromuscular diseases [C10.668] • Peripheral nervous system diseases [C10.668.829] . Molecular mimicry [G02.111.560]
(z.B. eine Durchwanderung im Rahmen einer Sinusitis) im Bereich der Geruchsrezeptoren oder aufgrund einer Reizleitungsstörung von olfaktorischen Bahnen auftreten. Virusinfektionen gelten als die häufigste Ursache einer solchen Reizleitungsstörung. Tierexperimentelle Studien konnten zeigen, dass die intranasale Inokulation von SARS-CoV-2 mit entzündlichen Zellinfiltraten und der Ausschüttung proinflammatorischer
Zytokine einhergeht. SARS-CoV-2-Virus-Antigene konnten dabei auch in ACE2(,angiotensin converting enzym $2^{\prime \prime}$ )-positiven olfaktorischen Nervenzellen immunhistochemisch nachgewiesen werden, sodass ein Neurotropismus postuliert wird [53]. Eine rezente Autopsiestudie berichtet über eine Akkumulation von Mikroglia und reaktiven Astrozyten als mögliches neuropathologisches Korrelat einer Anosmie [33]. 
Bezüglich der Behandlung einer Anosmie bzw. Ageusie gibt es aktuell keine evidenzbasierten Empfehlungen. Es gibt Hinweise darauf, dass eine orale oder topische Anwendung von Kortikosteroiden bei einer Anosmie durch Virusinfektion wirksam sein kann, indem sie Schleimhautentzündung und Ödeme reduziert [47]. Einer europäischen Studie zufolge wurden bei olfaktorischer Dysfunktion im Rahmen von COVID-19 empirisch nasale Kochsalzlösungsspülungen $(16,7 \%)$ sowie topische $(8,1 \%)$ oder orale Kortikosteroide $(2,5 \%)$ eingesetzt [25]. Mittlerweile wird Dexamethason in Deutschland zumindest bei schweren Verläufen einer COVID19 empfohlen [22], - wie der Effekt auf die Anosmie ist, bleibt abzuwarten.

\section{》) Bei der Mehrzahl der Patienten bilden sich die Symptome vollständig zurück}

Grundsätzlich ist die Prognose der Geruchs- oder Geschmackssinnstörungen als günstig anzusehen, denn bei der Mehrzahl der Patienten bilden sich die Symptome vollständig zurück [7]. Bei etwa einem Drittel der Patienten kommt es zu einer kompletten Erholung innerhalb von vier Wochen. Die meisten Patienten berichteten über ein Abklingen der Symptome nach durchschnittlich 11 Tagen [1].

Cave Anosmie und Ageusie bei COVID19:

- treten häufig und früh im Krankheitsverlauf auf;

- sind in der Regel innerhalb weniger Tage bis Wochen rückläufig;

- in der MRT können Schwellungen, bei Persistenz auch eine Atrophie der Bulbi olfactorii sichtbar sein;

- spezifische Therapieempfehlungen existieren bisher nicht.

\section{Affektion anderer Hirnnerven}

Mononeuropathien und Polyneuritiden der Hirnnerven nach SARS-COV2Infektion wurden bisher vereinzelt berichtet. Beeinträchtigungen wurden für den N. opticus [50], N. oculomotorius [3
11 15], den N. abducens [11 13], N. facialis [17 21] und auch für die kaudalen Hirnnerven beschrieben [2, 30]. Während erstere schon nach wenigen Tagen rückläufig waren, persistierten bulbäre Symptome länger. In der MRT kann eine Schwellung einzelner Nerven beobachtet werden [36]. Therapeutisch wurde u.a. Chloroquin, Hydroxychloroquin oder intravenösen Immunglobulinen (IVIg; bei Verdacht auf ein atypisches bzw. inkomplettes Miller Fisher-Syndrom) verabreicht. Diese Ansätze spiegeln teilweise Empfehlungen wider, die nicht mehr gültig sind.

Ähnlich wie beim Guillain-BarréSyndrom wird auch bei Hirnnervenparesen neben einer direkten Infektion (Neurotropismus) eine autoimmune Genese diskutiert. Für die erste Hypothese sprechen Autopsiebefunde COVID-19 verstorbener Patienten, bei denen SARSCoV-2-Proteine in Perikarya der Hirnnerven IX und $\mathrm{X}$ nachgewiesen wurden [33].

\section{Guillain-Barré-Syndrom und Miller Fisher-Syndrom}

Das Konzept zur Pathogenese des Guillain-Barré-Syndroms (GBS) als Prototyp einer postinfektiösen autoimmunen Neuropathie [43] sowie Fallberichte und Fallserien aus besonders von der Pandemie betroffenen Ländern wie Spanien [10], Italien [14], Indien [35] und den USA [8] lassen zum aktuellen Zeitpunkt eine Kausalität eines GBS nach einer SARS-COV-Infektion plausibel erscheinen.

I) Nach einer Influenza scheint das Risiko für GBS deutlich größer zu sein als nach COVID-19

Aussagekräftige, prospektiv erhobene Daten aus Fallkontroll- bzw. Kohortenstudien, die den Zusammenhang eines GBS mit einer COVID-19-Erkrankung bestätigen und deren Häufigkeit bestimmen könnten, liegen allerdings nicht vor. Lediglich aus Norditalien werden (retrospektiv) erhobene, epidemiologische Daten mitgeteilt, die für die Pandemie von Februar bis April 2020 eine 2,6- fach höhere Inzidenz für ein GBS angeben [14]. In einer gerade publizierten Studie aus Großbritannien, die teilweise retrospektiv, teilweise prospektiv Daten erhob, zeigte sich hingegen keine vermehrte Häufigkeit eines GBS während der ersten Infektionswelle in Großbritannien [20]. Aus Bergamo wurde hingegen berichtet, dass von 1760 COVID-19Patienten, die zwischen dem 23.02. und 30.04.2020 im dortigen Papa Giovanni XXIII-Hospital aufgenommen wurden, 17 Patienten (ca. 1\%) ein GBS hatten [40]. Vergleiche mit anderen populationsbasierten Datensätzen beim GBS lassen den Schluss zu, dass ein GBS nach COVID-19 offenbar deutlich seltener ist als beispielsweise nach einer Zika-VirusInfektion (bis zu $63 \%$ aller hospitalisierten Patienten; [4]), aber möglicherweise häufiger auftritt als nach einer Infektion mit Varizella-Zoster-Virus (0,01\%; [19 27]). Auch nach einer Influenza scheint das Risiko an einem GBS zu erkranken deutlich größer zu sein als nach COVID19. Tam und Kollegen berechnet ein 18fach erhöhtes Risiko für GBS nach grippeähnlichen Erkrankungen [49]. Bei bis zu $14 \%$ von GBS-Patienten finden sich serologisch Hinweise für eine kürzlich aufgetretene Influenza-A-Infektion [28].

\section{》) Das Intervall zwischen COVID-19-Symptomen und dem Beginn eines GBS ist kurz}

Aus den bisher publizierten klinischen Daten lässt sich ableiten, dass die meisten Patienten, die an einem GBS nach COVID-19 erkrankten, ein „klassisches GBS“ mit Zeichen der Demyelinisierung in der Elektroneurographie und eine zytoalbuminäre Dissoziation im Liquor aufwiesen. Anti-Gangliosid-Antikörper wurden nur ganz vereinzelt nachgewiesen. GBS-Varianten wie AMAN (akute motorische axonale Neuropathie) oder die „Ropper-Variante“ [21] wurden zwar auch, aber deutlich seltener beobachtet. Bei ungefähr jedem 3. Patienten trat eine Ateminsuffizienz auf und bei $40 \%$ der Patienten wurde eine Aufnahme auf die Intensivstation aufgrund des GBS erforderlich. Post-COVID-19-GBS-Patienten scheinen auf die übliche Behandlung 
Hier steht eine Anzeige.

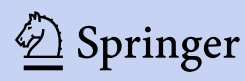


eines GBS mit IVIg bzw. Plasmapherese anzusprechen.

Auffällig ist bei vielen Patienten ein relativ kurzes Intervall zwischen Symptomen einer SARS-CoV-2-Infektion und Beginn eines GBS [48]. Bei anderen viralen Triggern eines GBS tritt dieses häufig mit einer Latenz von 2 bis 4 Wochen nach der durchgemachten Infektion auf [29]. In der aktuell größten Fallserie von 30 COVID-19-Patienten, die an einem GBS erkrankten, waren bei $>80 \%$ der Patienten bei Beginn des GBS noch COVID-19-Symptome vorhanden [14]. Diese Beobachtung findet sich auch in anderen Fallserien und wirft die Frage auf, ob es sich eher um ein para- und nicht um ein postinfektiöses Geschehen handelt. Dagegen sprechen allerdings gleich mehrere Gründe:

1. Die (asymptomatische) Inkubationszeit der SARS-CoV-2-Infektion kann bis zu 14 Tage betragen, was die Berechnung des Zeitintervalls zwischen Infektion und Entwicklung des GBS erschwert.

2. In der klinischen Praxis, zumal bei strikt isolierten oder beatmungspflichtigen Patienten, ist es häufig schwierig, eine subakute Lähmung frühzeitig zu erkennen. Dies könnte auch ein Grund sein, warum GBS bei COVID-19-Patienten unterdiagnostiziert wird und somit eine klare Assoziation „maskiert“.

3. Neuropathologisch gibt es zudem zum aktuellen Zeitpunkt keine gesicherten Erkenntnisse, ob SARS-CoV-2 direkt periphere Nerven(wurzeln) infizieren kann.

In Liquoruntersuchungen, die auch bei Post-COVID-19-GBS die klassische „GBS-Konstellation“ einer zytoalbuminären Dissoziation (ohne Liquorpleozytose) zeigte, wurde das Virus bisher nicht nachgewiesen.

Merke Bei schweren COVID-19-Verläufen und Beatmungspflichtigkeit oder prolongiertem „Weaning“ sollte ein GBS als Differenzialdiagnose berücksichtigt werden.

Neben GBS wurden auch mehrere Fälle eines Miller Fisher-Syndroms (MFS) bei SARS-CoV-2-positiven Patienten be- richtet [4]. Auffallend ist, dass oft keine Anti-Gangliosid-Antikörper im Serum nachweisbar waren. Diese haben normalerweise eine Sensitivität von $>90 \%$.

Die bisherigen Daten einer SARSCoV-2-Infektion mit einem konsekutiven GBS implizieren, dass bei Verdacht auf ein GBS eine Diagnostik hinsichtlich einer vorangegangen und möglicherweise noch aktiven SARS-CoV-2-Infektion erfolgen sollte.

In Einzelfällen wurde auch über eine Armplexopathie in Zusammenhang mit COVID-19 berichtet [37].

Cave GBS/MFS bei SARS-CoV2-Infektion:

- ist bisher selten beschrieben worden;

- epidemiologisch ist ein Zusammenhang bisher nicht bewiesen;

- häufig sind bei Beginn noch Symptome einer COVID-19-Erkrankung vorhanden;

- es finden sich Demyelinisierungszeichen in der Elektroneurographie und

- eine zytoalbuminäre Dissoziation im Liquor (ohne SARS-COV2Nachweis);

- Antigangliosidantikörper sind in der Regel nicht vorhanden;

- eine respiratorische Insuffizienz ist häufig;

- spricht auf IVIg oder Plasmapherese an.

\section{Fazit für die Praxis}

- Anosmie bzw. Ageusie sind bei weitem die häufigsten Hirnnervenstörungen, die bisher bei COVID19 berichtet wurden. Andere Hirnnervenausfälle (N. facialis, Augenmuskelnerven) erscheinen deutlich seltener.

- Kontrovers ist bisher die Datenlage zum Guillain-Barré-Syndrom. Eine Kausalität, die durch die hohe Anzahl von Fallberichten und auch durch die Pathogenese der Erkrankung suggeriert wird, ist bisher noch nicht in größeren Studien überzeugend belegt worden.

\section{Korrespondenzadresse}

Prof. Dr. Helmar C. Lehmann

Klinik und Poliklinik für Neurologie,

Universitätsklinikum Köln

Kerpener Straße 62, 50937 Köln, Deutschland

helmar.lehmann@uk-koeln.de

\section{Einhaltung ethischer Richtlinien}

Interessenkonflikt. H.C. Lehmann, B. Schoser, G. Wunderlich, P. Berlit und G.R. Fink geben an, dass kein Interessenkonflikt besteht.

Für diesen Beitrag wurden von den Autoren keine Studien an Menschen oder Tieren durchgeführt. Für die aufgeführten Studien gelten die jeweils dort angegebenen ethischen Richtlinien.

\section{Literatur}

1. Amer MA, Elsherif HS, Abdel-Hamid AS, Elzayat $S$ (2020) Early recovery patterns of olfactory disorders in COVID-19 patients; a clinical cohort study. Am J Otolaryngol 41:102725. https://doi. org/10.1016/j.amjoto.2020.102725

2. Aoyagi Y, Ohashi M, Funahashi R, Otaka Y, Saitoh E (2020) Oropharyngeal Dysphagia and Aspiration Pneumonia Following Coronavirus Disease 2019: A Case Report. Dysphagia 35(4):545-548

3. Belghmaidi S, Nassih H, Boutgayout S et al (2020) Third cranial nerve palsy presenting with unilateral diplopia and strabismus in a 24-year-old woman with COVID-19. Am J Case Rep 21:e925897. https:// doi.org/10.12659/AJCR.925897

4. Brito Ferreira ML, de Militão de Albuquerque MFP, Brito CAA et al (2020) Neurological disease in adults with Zika and chikungunya virus infection in Northeast Brazil: a prospective observational study. Lancet Neurol 19:826-839. https://doi.org/ 10.1016/S1474-4422(20)30232-5

5. Chiu A, Fischbein N, Wintermark M et al (2020) COVID-19-induced anosmia associated with olfactory bulb atrophy. Neuroradiology. https:// doi.org/10.1007/s00234-020-02554-1

6. Cummings MJ, Baldwin MR, Abrams D et al (2020) Epidemiology, clinical course, and outcomes of critically ill adults with COVID-19 in New York City: a prospective cohortstudy. Lancet 395:1763-1770. https://doi.org/10.1016/S0140-6736(20)31189-2

7. D'Ascanio L, Pandolfini M, Cingolani $C$ et al (2020) Olfactory dysfunction in COVID-19 patients: prevalence and prognosis for recovering sense of smell. Otolaryngol Head Neck Surg. https://doi. org/10.1177/0194599820943530

8. Defabio AC, Scott TR, Stenberg RT, Simon EL (2020) Guillain-Barré syndrome in a patient previously diagnosed with COVID-19. Am J Emerg Med. https://doi.org/10.1016/j.ajem.2020.07.074

9. Dhont S, Callens R, Stevens Det al (2020) Myotonic dystrophy type 1 as a major risk factor for severe COVID-19? Acta Neurol Belg. https://doi.org/10. 1007/s13760-020-01514-z

10. Diez-Porras L, Vergés E, Gil F et al (2020) GuillainBarré-Strohl syndrome and COVID-19: Case report and literature review. Neuromuscul Disord 30:859-861. https://doi.org/10.1016/j.nmd.2020. 08.354 
11. Dinkin M, Gao V, Kahan J et al (2020) COVID-19 presenting with ophthalmoparesis from cranial nerve palsy. Neurology 95:221-223. https://doi org/10.1212/WNL.0000000000009700

12. Ellul MA, Benjamin L, Singh B et al (2020) Neurological associations of COVID-19. Lancet Neurol 19:767-783. https://doi.org/10.1016/ S1474-4422(20)30221-0

13. Falcone MM, Rong AJ, Salazar H et al (2020) Acute abducens nerve palsy in a patient with the novel coronavirus disease (COVID-19). J AAPOS. https:// doi.org/10.1016/j.jaapos.2020.06.001

14. Filosto M, Cotti Piccinelli S, Gazzina $S$ et al (2020) Guillain-Barré syndrome and COVID-19: an observational multicentre study from two Italian hotspot regions. J Neurol Neurosurg Psychiatry. https://doi.org/10.1136/jnnp-2020-324837

15. Fitzpatrick JC, Comstock JM, Longmuir RA et al (2020) Cranial Nerve III Palsy in the setting of COVID 19 Infection. J Neuroophthalmol. https://doi.org/ 10.1097/WNO.0000000000001160

16. Giacomelli A, Pezzati L, Conti F et al (2020) Self-reported olfactory and taste disorders in patients with severe acute respiratory Coronavirus 2 infection: a cross-sectional study. Clin Infect Dis 71:889-890. https://doi.org/10.1093/cid/ciaa330

17. Homma $Y$, Watanabe $M$, Inoue K, Moritaka T (2020) Coronavirus disease-19 pneumonia with facial nerve palsy and olfactory disturbance. Intern Med 59:1773-1775. https://doi.org/10. 2169/internalmedicine.5014-20

18. Huang C, Wang Y, Li X et al (2020) Clinical features of patients infected with 2019 novel coronavirus in Wuhan, China. Lancet 395:497-506. https://doi. org/10.1016/S0140-6736(20)30183-5

19. Kang J-H, Sheu J-J, Lin H-C (2010) Increased risk of Guillain-Barré Syndrome following recent herpes zoster: a population-based study across Taiwan. Clin Infect Dis 51:525-530. https://doi.org/10. $1086 / 655136$

20. Keddie S, Pakpoor J, Mousele C et al (2020) Epidemiological and cohort study finds no association between COVID-19 and Guillain-Barre syndrome. Brain. https://doi.org/10.1093/brain/ awaa433

21. Khaja M, Gomez GPR, Santana Y et al (2020) A 44-year-old hispanic man with loss of taste and bilateral facial weakness diagnosed with GuillainBarré syndrome and Bell's palsy associated with SARS-coV-2 infection treated with intravenous immunoglobulin. Am J Case Rep 21:e927956. https://doi.org/10.12659/AJCR.927956

22. Kluge $S$, Janssens $U$, Spinner CD, Pfeifer $M$, Marx G, Karagiannidis C; Guideline group (2021) Clinical Practice Guideline: Recommendations on Inpatient Treatment of Patients with COVID-19. Dtsch Arztebl Int. https://doi.org/10.3238/arztebl. m2021.0110. Epub ahead of print. PMID: 33531113

23. Koc G, Odabasi Z, Tan E (2020) Myasthenic syndrome caused by hydroxychloroquine used for COVID-19 prophylaxis. J Clin Neuromuscul Dis 22:60-62. https://doi.org/10.1097/CND. 0000000000000316

24. Laurendon T, Radulesco T, Mugnier J et al (2020) Bilateral transient olfactory bulb edema during COVID-19-related anosmia. Neurology 95:224-225. https://doi.org/10.1212/WNL. 0000000000009850

25. Lechien JR, Chiesa-Estomba CM, De Siati DR et al (2020) Olfactory and gustatory dysfunctions as a clinical presentation of mild-to-moderate forms of the coronavirus disease (COVID-19): a multicenter European study. Eur Arch Otorhinolaryngol
277:2251-2261. https://doi.org/10.1007/s00405020-05965-1

26. Lehmann HC, Burke D, Kuwabara S (2019) Chronic inflammatory demyelinating polyneuropathy: update on diagnosis, immunopathogenesis and treatment. J Neurol Neurosurg Psychiatry 90:981-987. https://doi.org/10.1136/jnnp-2019320314

27. Lehmann HC, Hartung H-P (2010) Varicella-zoster virus: another trigger of Guillain-Barré syndrome? Clin Infect Dis 51:531-533. https://doi.org/10. $1086 / 655137$

28. Lehmann HC, Hartung H-P, Kieseier BC, Hughes RAC (2010) Guillain-Barré syndrome after exposure to influenza virus. Lancet Infect Dis 10:643-651. https://doi.org/10.1016/S1473-3099(10)70140-7

29. Lehmann HC, Hughes RAC, Kieseier BC, Hartung H-P (2012) Recent developments and future directions in Guillain-Barré syndrome. J Peripher Nerv Syst 17(Suppl 3):57-70. https://doi.org/10. 1111/j.1529-8027.2012.00433.x

30. Liberatore G, De Santis T, Doneddu PE, Gentile F, Albanese A, Nobile-Orazio E (2020) Clinical Reasoning: A case of COVID-19-associated pharyngeal-cervical-brachial variant of GuillainBarré syndrome. Neurology 95(21):978-983

31. Luers JC, Rokohl AC, Loreck N et al (2020) Olfactory and gustatory dysfunction in Coronavirus disease 19 (COVID-19). Clin Infect Dis. https://doi.org/10. 1093/cid/ciaa525

32. Lv H, Zhang W, Zhu Z et al (2020) Prevalence and recovery time of olfactory and gustatory dysfunction in hospitalized patients with COVID19 in Wuhan, China. Int J Infect Dis 100:507-512. https://doi.org/10.1016/j.ijid.2020.09.039

33. Matschke J, Lütgehetmann $M$, Hagel $C$ et al (2020) Neuropathology of patients with COVID19 in Germany: a post-mortem case series. Lancet Neurol 19:919-929. https://doi.org/10. 1016/S1474-4422(20)30308-2

34. Mehraeen E, Behnezhad F, Salehi MA et al (2020) Olfactory and gustatory dysfunctions due to the coronavirus disease (COVID-19): a review of current evidence. Eur Arch Otorhinolaryngol. https://doi. org/10.1007/s00405-020-06120-6

35. Nanda S, Handa R, Prasad A et al (2020) Covid-19 associated Guillain-Barre Syndrome: contrasting tale of four patients from a tertiary care centre in India. Am J Emerg Med. https://doi.org/10.1016/j. ajem.2020.09.029

36. Pan S, Chen WC, Baal JD, Sugrue LP (2020) Neuroradiological features of mild and severe SARS-coV-2 infection. Acad Radiol 27:1507-1514. https://doi.org/10.1016/j.acra.2020.08.026

37. Paterson RW, Brown RL, Benjamin L et al (2020) The emerging spectrum of COVID-19 neurology: clinical, radiological and laboratory findings. Brain 143:3104-3120. https://doi.org/10.1093/brain/ awaa240

38. Radmard S, Epstein SE, Roeder HJ et al (2020) Inpatient neurology consultations during the onset of the SARS-coV-2 New York city pandemic a single center case series. Front Neurol 11:805. https://doi.org/10.3389/fneur.2020.00805

39. Rein N, Haham N, Orenbuch-Harroch E et al (2020) Description of 3 patients with myasthenia gravis and COVID-19. J Neurol Sci 417:117053. https:// doi.org/10.1016/j.jns.2020.117053

40. Rifino N, Censori B, Agazzi E et al (2020) Neurologic manifestations in 1760 COVID-19 patients admitted to Papa Giovanni XXIII Hospital, Bergamo, Italy. J Neurol. https://doi.org/10.1007/s00415-02010251-5
41. Schmidt J (2018) Current classification and management of inflammatory myopathies. J Neuromuscul Dis 5:109-129. https://doi.org/10. 3233/JND-180308

42. Schneider C, Wunderlich G, Bleistein J et al (2017) Lymphocyte antigens targetable by monoclonal antibodies in non-systemic vasculitic neuropathy. JNeurol Neurosurg Psychiatry 88:756-760. https:// doi.org/10.1136/jnnp-2017-315878

43. Shahrizaila N, Lehmann HC, Kuwabara S (2021) Guillain-Barré syndrome. Lancet. https://doi.org/ 10.1016/S0140-6736(21)00517-1.Epub ahead of print. PMID: 33647239

44. Siddiqui AK, Huberfeld SI, Weidenheim KM et al (2007) Hydroxychloroquine-induced toxic myopathy causing respiratory failure. Chest 131:588-590. https://doi.org/10.1378/chest.061146

45. Singh S, Govindarajan R (2020) COVID-19 and generalized myasthenia Gravis exacerbation: a case report. Clin Neurol Neurosurg 196:106045. https://doi.org/10.1016/j.clineuro.2020.106045

46. Stein M, Bell MJ, AngLC (2000)Hydroxychloroquine neuromyotoxicity. JRheumatol 27:2927-2931

47. Stenner M, Vent J, Hüttenbrink K-B et al (2008) Topical therapy in anosmia: relevance of steroidresponsiveness. Laryngoscope 118:1681-1686. https://doi.org/10.1097/MLG.0b013e31817c1368

48. Svačina MKR, Kohle F, Sprenger A, Lehmann HC (2021) Could symptom overlap of COVID-19 and Guillain-Barré syndrome mask an epidemiological association? J Neurol 17:1-3. https://doi.org/10. 1007/s00415-021-10515-8. Epub ahead of print. PMID: 33730264;PMCID:PMC7966909

49. Tam CC, O'Brien SJ, Petersen I et al (2007) Guillain-Barré syndrome and preceding infection with campylobacter, influenza and Epstein-Barr virus in the general practice research database. Plos One 2:e344. https://doi.org/10.1371/journal. pone. 0000344

50. Tisdale AK, Chwalisz BK (2020) Neuro-ophthalmic manifestations of coronavirus disease 19. Curr Opin Ophthalmol 31:489-494. https://doi.org/10. 1097/ICU.0000000000000707

51. Tsivgoulis G, Fragkou PC, Lachanis S et al (2020) Olfactory bulb and mucosa abnormalities in persistent COVID-19 induced anosmia: a magnetic resonance imaging study. Eur J Neurol. https://doi. org/10.1111/ene.14537

52. Vaira LA, Hopkins C, Petrocelli M et al (2020) Do olfactory and gustatory psychophysical scores have prognostic value in COVID-19 patients? A prospective study of 106 patients. J Otolaryngol Head Neck Surg 49:56. https://doi.org/10.1186/ s40463-020-00449-y

53. Zhang AJ, Lee AC-Y, Chu H et al (2020) SARS-CoV-2 infects and damages the mature and immature olfactory sensory neurons of hamsters. Clin Infect Dis. https://doi.org/10.1093/cid/ciaa995

54. Zhou F, Yu T, Du R et al (2020) Clinical course and risk factors for mortality of adult inpatients with COVID-19 in Wuhan, China: a retrospective cohort study. Lancet 395:1054-1062. https://doi.org/10. 1016/S0140-6736(20)30566-3 